\title{
Value of a Growth Mindset: Improving Endoscopy Coaching and Mentoring
}

\author{
Andrew Ming-Liang Ong ${ }^{1,2} \cdot$ Keith Siau $^{3}$
}

Accepted: 12 October 2021 / Published online: 27 October 2021

(c) The Author(s), under exclusive licence to Springer Science+Business Media, LLC, part of Springer Nature 2021

\begin{abstract}
The teaching of endoscopic procedures to trainees has radically changed from the previous "see one, do one, teach one" approach to the method termed "competency-based medical education" (CBME), which has transformed endoscopic training. Successful implementation of a CBME program requires learners to direct their own learning. Learners with a growth approach are more likely to thrive in such a system since they are learning-oriented and not performance-oriented. This method dictates that endoscopy mentors take a longer-term and broader view of the development of the trainee and are vital to cultivate a growth mindset in learners, while an endoscopy coach focuses on helping trainees apply principles of mastery learning and deliberate practice. The goal of the authors was to describe some principles of effective coaching and mentoring methods as applied to endoscopy training.
\end{abstract}

\section{Introduction}

One of the foremost challenges to teaching endoscopic skills is that, similar to any procedural skill, endoscopy requires both technical and cognitive skills [1]. Competency-based medical education (CBME) has transformed endoscopy training in that it places more emphasis on achieving milestones and the robust assessment of abilities rather than on procedural volume [2]. The successful implementation of CBME therefore requires learners to be partners in shaping their own growth through individualized, ongoing learning with the lifelong goal of technical mastery [3]. In other words, an individual's approach to learning will significantly affect their success in a CBME program.

Andrew Ming-Liang Ong

Andrew.ong.m.1@singhealth.com.sg

1 Singhealth Gastroenterology Residency Program, Singapore, Singapore

2 Department of Gastroenterology and Hepatology, Singapore General Hospital, 20 College Road, Level 3, Academia Building, Singapore 169856, Singapore

3 Department of Gastroenterology, Royal Cornwall Hospitals, Truro, UK

\section{Growth Mindset and Endoscopy}

Despite being in the same learning environment, why do some learners thrive while others struggle? Understanding the factors influencing this phenomenon is important, as mentors can then modify their approach to their learners in order to increase motivation and learning. "Growth mindset" is a motivation theory proposed by Carol Dweck [4], who posited that intelligence and the ability to change viewpoints can impact how one handles challenges, responds to criticism, and re-orients their goals. She also describes that individuals with a more fixed outlook believe that intelligence and ability are static and that success is derived from talent, whereas individuals with a more growth-oriented mindset believe these traits are malleable and success is largely derived from effort.

How can these approaches be applied to teaching endoscopy among trainees? Fixed-mindset learners behave in a manner that makes them 'look good' to reaffirm their selfworth and avoid failure, and therefore may choose easier endoscopy cases when in front of their supervisors for fear of appearing incompetent. In contrast, growth mindset learners focus on improving their competence in the pursuit of mastery and see failure as a part of the developmental process. They will be more willing to choose cases that are challenging, as they regard these cases as learning opportunities through seeking feedback for improvement. 
Wolcott et al. [5] cited several strategies that promote a growth mindset, including helping learners create learning goals focused on progress rather than performance goals. Improving the delivery of constructive feedback to learners that focuses on effort and observable behaviors instead of personality traits or talents was also a common strategy [5]. The challenge for many educators is to overcome years of training with a fixed approach to teaching due to rote memorization and standardized assessment practices [6]. Others have suggested that simply introducing learners to the rationale and practices for adopting a growth mindset can be effective [7]. We describe briefly two concepts in endoscopy training that encourage cultivating a growth mindset, namely mastery learning and deliberate practice. To implement these concepts effectively in a CBME system, we also describe why it is imperative for programs to have endoscopy coaches and mentors.

\section{Mastery Learning and Deliberate Practice}

Mastery learning stipulates that trainees must acquire foundational levels of knowledge, skills, and attitudes prior to progressing to more complicated tasks. Since learning should be rigorously measured and compared with fixed achievement standards without limiting the time needed to reach outcomes [8], endoscopic curriculum developers should thoughtfully plan specific, sequential learning experiences and defined milestones in order to achieve the desired standards.

The accurate measurement of reproducible performance in a well-defined task in endoscopy (e.g. torque control of colonoscope in the sigmoid colon) while providing performance data, ultimately improving specific aspects of performance through the provision of personalized feedback and "deliberate practice" by the trainee, defined as "the individualized training activities specially designed by a coach or teacher to improve specific aspects of an individual's performance through repetition and successive refinement." [9]

Despite the overall goal of all trainees reaching competency, trainees have different learning aptitudes. Since the beauty of mastery learning is manifest in the flexibility given to trainees to deliberately practice at their own pace until mastery of the task is achieved, this flexibility requires an individualized approach to training, and therefore reinforces the importance of the role of the endoscopy coach.

\section{Coaching in Endoscopy}

Spurred on by comparisons to the worlds of sports, music and executive coaching, medical educators have begun embracing the idea that coaching may effectively foster learning [10], an approach that seems even more intuitive for procedural specialties that encompass well-defined tasks such as surgery or endoscopy. Lovell's recent review of coaching in medical education [10] revealed good-quality data showing that coaching improves technical skills acquisition while reducing surgical error rates.

Practically speaking, the primary function of the endoscopy coach is to objectively assess the performance of endoscopy, often with the aid of direct observation competency assessment tools, providing performance-enhancing instruction and feedback. To achieve this goal, the coach must be able to deconstruct the individual tasks that comprise the overall procedure, verbally explaining how to perform specific maneuvers, such as step-by-step loop reduction techniques for a difficult sigmoid colon intubation, and lucidly troubleshoot difficulties while avoiding taking control of the endoscope. The coach would then need to be able to detect specific deficiencies, offering constructive advice in the form of performanceenhancing directive motor instruction during the procedure. Thereafter, it is important that the coach continuously observes the changes in technique, and if required, provide further corrective instruction. As part of the learning process, the trainee should be provided with opportunities to practice the suggested changes. The probability of success is enhanced if the coach fosters a working relationship with the trainee so as to create an open culture of honest feedback and trust.

An example of an application of coaching to guide mastery learning and deliberate practice was published recently [11], in which 6-week upper endoscopy training was provided for $1^{\text {st }}$ year gastroenterology fellows using the principles of simulation-based mastery learning along with real-time virtual coaching in order to overcome logistical issues stemming from the ongoing pandemic. The procedure of upper endoscopy was deconstructed into smaller observable tasks such as tip deflection, which were practiced by the trainees repeatedly in isolation. Throughout their training, the trainees were directly observed by a dedicated instructor who could provide specific constructive feedback according to well-defined performance standards. The trainees were then given opportunities to practice the specific deficiencies identified after each feedback session, thus promoting the idea of deliberate practice. Only when they had reached a minimum competency of these tasks were they exposed to sequentially more difficult tasks such as using biopsy forceps or hemostatic clipping. Even 2 months after the study, the majority of trainees were still using the techniques they were taught during this course.

\section{Endoscopy Mentors}

Since often used synonymously, there is understandable confusion between the terms "mentor" and "coach", especially in the case of procedural teaching. Despite the seeming 
overlap between these terms, there are distinct differences in purpose, skills required, and instructional strategies. Though when teaching faculty are in short supply, faculty may function as both, differentiating the two teaching functions is important, requiring training programs to train their faculties appropriately. Broadly speaking, a mentor, sometimes also referred to as an academic advisor or supervisor, takes a longer-term and more expansive view of the development of the trainee, whilst the coach focuses on helping trainees gain insights into their own assumptions, clarify the meaning of relevant outcomes, and help identify specific actions needed to achieve a desired result [12].

If the program has the luxury of having endoscopy mentors in addition endoscopy coaches, the mentor can complement the endoscopy coaches by providing a longer-term and more global approach. Both mentors and coaches should be fully aware of the training requirements and milestones, while having a sound knowledge of the motivations and learning styles of the trainee in order to facilitate individualization of the learning plan. Being mindful of the learner's outlook to learning (e.g. fixed vs growth mindset) is useful for the mentor to guide the growth of the learner. Proposed benefits of the growth mindset orientation [5] include helping learners to be more receptive to feedback, supporting collaborative relationships between learners and educators, as well as positive impacts on resiliency, perseverance, and psychological well-being that foster safer learning environments, which are all important for acquiring knowledge and skills. Having a growth mindset will also lead to constant self-reflection in the trainee, and is a key first step in improvement. Since trainees are not always able to identify their deficits or learning needs [13], mentors must help guide their self-reflection. If there is evidence that the learner has a fixed mindset approach to learning endoscopy, the mentor should explicitly explore this with the learner. Once deficiencies have been identified by both the mentor and learner about their procedural competency, they should jointly collaborate so as to develop an individualized learning plan with outcomes that are specific, actionable, and time-bound. Tailored learning activities can then be planned with the purpose of achieving these outcomes. An example would be direct observation of colonoscopies and weekly assessments over a three-month period. The mentor should communicate with the endoscopy coach on the agreed plan, preferably in a shared written document.

\section{What Are the Principles of Coaching Well?}

In order to understand the essential elements of successful coaching, Watling et al. [14] asked clinician teachers to describe their experiences as coaches while articulating principles that successfully guided their coaching approach. Their findings suggest that successful coaching requires (1) mutual engagement, (2) reflection and (3) embracing failure.

Good endoscopy coaches are therefore not distinguished by their knowledge and skill but rather by their ability to motivate, inspire, and bring out the best in their trainees. Unlike teaching, which is often driven by the teacher's agenda, and mentoring, which is driven by the mentee's needs, coaching is regarded as a mutual partnership oriented towards growth and development [14]. A good coach will know their trainee, their background, personality, cognitive capacity, and training preferences. Moreover, they may look out for their physical and mental well-being, encouraging scholarly activity outside of the endoscopy room. Good coaches should also be able to juggle directive endoscopy instruction versus guiding trainees to reflect on what was and was not successful. The proactive use of reflection can also empower learners to maintain progress between coaching sessions. Good coaches also know how to destigmatize failure by being explicit about its inevitability, and therefore enable trainees to learn from mistakes within the constraints of patient safety, which can then be used for personal reflection and development, bearing in mind that achieving the correct balance of psychologically safe coaching while providing optimal patient care is challenging.

\section{Faculty Development of Endoscopy Trainers}

Though in disciplines such as sports and music, welldefined pathways exist to guide coaches, in medicine, fellow training by faculty is often taken for granted, partially due to the many functions of teaching faculty, including as supervisors, assessors, and doctors. Wolff et al. [15] delineated specific competencies for coaching in medical education that broadly included (1) coaching structure and process, (2) relational skills, (3) coaching skills, (4) coaching theories, and (5) coaching development. Programs may use these competencies in order to design faculty development programs for their coaches.

With regards to endoscopy coaching, faculty should design learning activities that incorporate the concepts of mastery learning, deliberate practice, and effective delivery of feedback. Trainers should be taught how to observe endoscopic procedures so that high-quality constructive feedback can be offered. Conversely, the principles of mastery learning may also be applied to faculty members, who themselves may benefit from peer-reviewed observation, assessment, and constructive feedback regarding their coaching techniques. 


\section{Conclusion}

High-level endoscopy is not a natural talent but rather can be trained with deliberate practice. Cultivating a growth mindset in procedural learning should be the ultimate goal of endoscopy trainers so that learners are able to evaluate their own performance, design their own learning goals, and then increasingly monitor and refine their own technique. Coaching and mentoring, if done well and purposefully, can be the engine that drives this.

\section{References}

1. Sewell JL, Bowen JL, Ten Cate O, O'Sullivan PS, Shah B, Boscardin CK. Learning Challenges, Teaching Strategies, and Cognitive Load: Insights from the Experience of Seasoned Endoscopy Teachers. Acad Med. 2020;95:794-802. https://doi.org/10.1097/ ACM.0000000000002946.

2. Northup PG, Argo CK, Muir AJ, DeCross AJ, Coyle WJ, Oxentenko AS. Procedural Competency of Gastroenterology Trainees: From Apprenticeship to Milestones. Gastroenterology. 2013;144:677-680. https://doi.org/10.1053/J.GASTRO.2013. 02.020 .

3. Richardson D, Kinnear B, Hauer KE et al. Growth mindset in competency-based medical education. Med Teach. 2021;43:751757. https://doi.org/10.1080/0142159X.2021.1928036.

4. Dweck, C. S. (2006). Mindset: The new psychology of success. Random House

5. Wolcott MD, McLaughlin JE, Hann A et al. A review to characterise and map the growth mindset theory in health professions education. Med Educ. 2021;55:430-440. https://doi.org/10.1111/ medu. 14381

6. Shapiro N, Dembitzer A. Faculty development and the growth mindset. Med Educ. 2019;53:958-960. https://doi.org/10.1111/ MEDU.13948.
7. Klein J, Delany C, Fischer MD, Smallwood D, Trumble S. A growth mindset approach to preparing trainees for medical error. BMJ Qual Saf. 2017;26:771-774. https://doi.org/10.1136/ BMJQS-2016-006416.

8. McGaghie WC. Mastery learning: It is time for medical education to join the 21st century. Acad Med. 2015;90:1438-1441. https:// doi.org/10.1097/ACM.0000000000000911.

9. Ericsson KA. Acquisition and maintenance of medical expertise: A perspective from the expert-performance approach with deliberate practice. Acad Med. 2015;90:1471-1486. https://doi.org/10. 1097/ACM.0000000000000939.

10. Lovell B. What do we know about coaching in medical education? A literature review. Med Educ. 2018;52:376-390. https://doi.org/ 10.1111/MEDU.13482.

11. Soetikno R, Cabral-Prodigalidad PA, Kaltenbach T. SimulationBased Mastery Learning With Virtual Coaching: Experience in Training Standardized Upper Endoscopy to Novice Endoscopists. Gastroenterology 2020;159:1632-1636. https://doi.org/10.1053/j. gastro.2020.06.096.

12. Deiorio NM, Carney PA, Kahl LE, Bonura EM, Juve AM. Coaching: a new model for academic and career achievement. Med Educ Online. 2016;21:33480. https://doi.org/10.3402/MEO.V21.33480.

13. Eva KW, Regehr G. I'll never play professional football and other fallacies of self-assessment. J Contin Educ Health Prof. 2008;28:14-19. https://doi.org/10.1002/CHP.150.

14. Watling CJ, LaDonna KA. Where philosophy meets culture: exploring how coaches conceptualise their roles. Med Educ. 2019;53:467-476. https://doi.org/10.1111/medu.13799.

15. Wolff M, Deiorio NM, Miller Juve A et al. Beyond advising and mentoring: Competencies for coaching in medical education. Med Teach. 2021. https://doi.org/10.1080/0142159X.2021.1947479.

Publisher's Note Springer Nature remains neutral with regard to jurisdictional claims in published maps and institutional affiliations. 